This item was submitted to Loughborough's Research Repository by the author.

Items in Figshare are protected by copyright, with all rights reserved, unless otherwise indicated.

The effect of ion pumps on the speed of travelling waves in the fire-diffusefire model of $\mathrm{Ca} 2+$ release

PLEASE CITE THE PUBLISHED VERSION

LICENCE

CC BY-NC-ND 4.0

REPOSITORY RECORD

Coombes, S.. 2019. "The Effect of Ion Pumps on the Speed of Travelling Waves in the Fire-diffuse-fire Model of Ca2+ Release". figshare. https://hdl.handle.net/2134/750. 


\title{
The effect of ion pumps on the speed of travelling waves in the fire-diffuse-fire model of $\mathrm{Ca}^{2+}$ release
}

\author{
S CoOmbes* \\ Nonlinear and Complex Systems Group, Department of Mathematical Sciences, \\ Loughborough University, Loughborough, Leicestershire, LE11 3TU, UK
}

\begin{abstract}
The fire-diffuse-fire model provides an idealised model of $\mathrm{Ca}^{2+}$ release within living cells. The effect of calcium pumps, which drive $\mathrm{Ca}^{2+}$ back into internal stores, is often neglected for mathematical simplicity. Here we show how to explicitly analyse such effects by extending the work of Keizer et al. (J. E. Keizer, G. D. Smith, S. Ponce Dawson and J. Pearson, 1998, Saltatory propagation of $\mathrm{Ca}^{2+}$ waves by $\mathrm{Ca}^{2+}$ sparks, Biophysical Journal, 75, 595-600). For travelling waves, in which release events occur sequentially, we construct the speed of waves in terms of the time-scale at which pumps operate. An immediate consequence of this analysis is that the inclusion of calcium pumps leads to multiple solutions. A linear stability analysis determines those solution branches in parameter space which are stable. Numerical continuation is used to provide explicit examples of the bifurcation diagrams of the speed of waves as a function of physiologically significant system parameters.
\end{abstract}

\section{Introduction}

The fire-diffuse-fire (FDF) model of Keizer et al. 1998 (see also Pearson and Ponce Dawson, 1998, Ponce Dawson et al., 1999]) is relevant to the study of travelling wave behaviour observed when $\mathrm{Ca}^{2+}$ is released from internal stores in living cells. $\mathrm{Ca}^{2+}$ is stored intracellularly in the endoplasmic or sarcoplasmic reticulum at 2-3 orders of magnitude greater than its concentration in the cytosol and is released by a nonlinear feedback process referred to as calciuminduced calcium release (CICR). CICR involves $\mathrm{Ca}^{2+}$ release through $\mathrm{Ca}^{2+}$ channels that are receptors for $\mathrm{IP}_{3}$ (inositol $(1,4,5)$-trisphosphate). These channels are activated at slightly elevated levels of cytosolic $\mathrm{Ca}^{2+}$ and then inactivated as the level of $\mathrm{Ca}^{2+}$ rises further. This mechanism for generating oscillations in

*S.Coombes@Lboro.ac.uk 
the concentration of cytosolic free $\mathrm{Ca}^{2+}$ is believed to underlie the waves that propagate as intra and intercellular waves over distance as large as $1 \mathrm{~mm}$ with speeds of between 5 and $20 \mu \mathrm{ms}^{-1}$ (for a review see Rooney and Thomas [1993]). When coupled to the kinetics of $\mathrm{Ca}^{2+}$ reuptake into stores (by $\mathrm{Ca}^{2+}$ ATPases), the CICR mechanism can lead to excitability and oscillations. Initiation sites or hot spots have been found to spontaneously release $\mathrm{Ca}^{2+}$ and can trigger a wave that spreads via the CICR process Parker and Yao, 1991]. Burst-like wave behaviour is commonly seen in immature Xenopus oocytes and in cardiac myocytes where saltatory waves propagate with a non-constant shape (see for example Callamaras et al. [1998]). Importantly, long ranged $\mathrm{Ca}^{2+}$ waves are capable of synchronising the activities of different cytoplasmic regions of a single cell, such as cortical granule exocytosis after egg fertilisation. In contrast to a saltatory wave, these waves have a continuous nature. Both types of wave are thought to be supported by the same mechanism, namely CICR. The existence of such long range spatial and temporal signalling by $\mathrm{Ca}^{2+}$ is one of the most significant findings of the last decade in the field of intracellular signalling and has stimulated many experiments for the elucidation of the role of $\mathrm{Ca}^{2+}$ waves in cell regulation (see for example Berridge and Dupont [1994], Berridge [1997, 1998], Bootman et al. [1997]). Theoretical and numerical studies of detailed biophysical models (see for example Snevd et al. [1993]. DuPont and Goldbeter [1994].J Jafri and Keizer [1995], Goldbeter [1996]) have also been performed and motivate the FDF model discussed in this paper. A major success of the FDF model is the natural description of both saltatory and continuous travelling waves.

The FDF model incorporates descriptions of the two major fluxes between the endoplasmic reticulum and the cytosol. The first is due to a pump which drives the $\mathrm{Ca}^{2+}$ up the gradient from the cytosol back into the endoplasmic reticulum and the second arises when the $\mathrm{IP}_{3}$ receptor/calcium channel opens and causes a large flux from the endoplasmic reticulum into the cytosol. After an open channel closes via inactivation, it cannot reopen for some time during which it is in a refractory state. Thus the release of $\mathrm{Ca}^{2+}$ by intracellular stores is self-regulating. Such events are commonly referred to as $\mathrm{Ca}^{2+}$ puffs or sparks and are the elementary events underlying $\mathrm{Ca}^{2+}$ waves. There are a variety of kinetic schemes that have been proposed to explain the detailed mechanism by which $\mathrm{Ca}^{2+}$ oscillations occur. Some assume that $\mathrm{IP}_{3}$ is necessarily oscillatory (for a discussion of beth oscillatory and non-oscillatory $\mathrm{IP}_{3}$ models see De Young and Keizer, 1992]), whilst others assume the existence of two pools for $\mathrm{Ca}^{2+}$ and emphasize the effect of CICR (e.g. DuPont and Goldbeter, 1994]). For a discussion of $\mathrm{IP}_{3}$ induced $\mathrm{Ca}^{2+}$ oscillations and an anology with the HodgkinHuxley the ry for electrical excitability in neurons see the review paper by $\mathrm{Li}$ et al. [1995]. The FDF model provides a caricature of $\mathrm{Ca}^{2+}$ release events that allows one to study an array of $\mathrm{Ca}^{2+}$ release sites that can interact via diffusion of $\mathrm{Ca}^{2+}$ and the triggering of a CICR like mechanism.

The one dimensional FDF model consists of a regular array of point-source release sites with lattice spacing $d$, embedded in a continuum in which calcium 
ions diffuse. Denoting the concentration of $\mathrm{Ca}^{2+}$ ions by $u(x, t)$ the FDF model is given by the following partial differential equation

$$
\frac{\partial u}{\partial t}=-\frac{u}{\tau_{d}}+D \frac{\partial^{2} u}{\partial x^{2}}+\sum_{n=-\infty}^{\infty} \delta\left(x-x_{n}\right) f\left(t-t_{n}\right), \quad x \in \mathbb{R}, t>0 .
$$

Note that $x_{n}=n d$ is the location of the $n$th $\mathrm{Ca}^{2+}$ release site and the first time that $u\left(x_{n}, t\right)$ crosses a threshold $u_{c}$ from below is denoted by $t_{n}$. Hence, the FDF model assumes that the site at $x_{n}$ releases only one $\mathrm{Ca}^{2+}$ puff at a time, $t_{n}$, determined by

$$
t_{n}=\inf \left\{t \mid u\left(x_{n}, t\right)>u_{c}, \frac{\partial u\left(x_{n}, t\right)}{\partial t}>0\right\} .
$$

The function $f(t)$ describes the shape of the $\mathrm{Ca}^{2+}$ puff and is considered as a rectangular pulse-shape given by

$$
f(t)=\frac{\sigma}{\tau_{R}} \Theta(t) \Theta\left(\tau_{R}-t\right)
$$

where $\Theta(x)$ is a step function $(\Theta(x)=0$ for $x<0, \Theta(x)=1$ for $x \geq 0), \sigma$ is the strength of the calcium puff and $\tau_{R}$ is the duration of $\mathrm{Ca}^{2+}$ release. The extension of the model to higher dimensions will not be considered here.

The inclusion of the decay time $\tau_{d}$ in (1) models the time-scale associated with the action of the pumps that resequester the $\mathrm{Ca}^{2+}$ back into the stores. Under normal physiological conditions pumps that are embedded in the membrane consume nucleotide trisphosphates and pump the $\mathrm{Ca}^{2+}$ up the gradient from the cytosol back into the stores. The effect of this term has been neglected in previous studies and can only be justified if the pumps operate on some very slow timescale. In this paper we focus on the effects of the decay term in (1) and show that it leads to the co-existence of travelling wave states with the same speed but differing stability. Moreover, the effect of such pumps on the speed, and indeed existence, of stable travelling waves can be significant.

In the absence of any sources $(\sigma=0)$ equation (1) is equivalent to the cable equation that is often used to model the spread of voltage in a passive unbranched dendritic cable. In fact, in the limit of zero lattice spacing, the FDF model has certain similarities with a recent model of dendritic cable with active spines due to Coombes and Bressloff [2000]. In this continuum model voltage spikes in compartments (spine-heads) electrically connected by an ohmic resistance to a dendritic cable can induce currents in neighboring spines via the spread of voltage along the cable. If the induced current is sufficiently large a spike or action potential may be generated. A sequence of such events at neighbouring spines generates a travelling wave. This model might indeed be considered as a spike-diffuse-spike model (SDS). Both FDF and SDS type systems are capable of composing global signals (travelling waves) from elementary events (puffs or spikes). 
In $\S 2$ we introduce the ansatz for constructing travelling wave solutions in the FDF model in terms of the release times of the first $\mathrm{Ca}^{2+}$ puffs at the sites occupied by calcium stores. We show that the travelling waves do not propagate with a constant profile. All quantities of interest can be related to the convolution of the Green's function of the well known cable equation with the shape of the $\mathrm{Ca}^{2+}$ puff generated by the CICR process. We explicitly evaluate this convolution and show how to generalise previous studies of the FDF model. The speed of travelling waves is constructed as a function of system parameters and it is seen that a finite decay term $\tau_{d}^{-1} \neq 0$ can lead to co-existence of solutions. Another consequence of the decay term is the possibility of propagation failure (of the single release saltatory wave) for small $\tau_{d}$. Furthermore, we present a linear stability analysis that can be used to ascertain that the faster of the travelling waves is physiologically relevant. In $\S 3$ we discuss the continuum limit of the FDF model in which travelling pulses can propagate with constant profile. Similar observations regarding stability are made. Finally in $\S 4$ we discuss possible extensions of this work as well as a generalisation of the FDF model that incorporates the effects of multiple release events.

\section{Travelling waves}

The solution of (1) can be expressed in terms of the Green's function of the cable equation as

$$
u(x, t)=\frac{\sigma}{\tau_{R}} \sum_{n=-\infty}^{\infty} \int_{t_{n}}^{t_{n}+\tau_{R}} \mathrm{~d} t^{\prime} G\left(x-x_{n}, t-t^{\prime}\right)
$$

where

$$
G(x, t)=\frac{\mathrm{e}^{-t / \tau_{d}}}{\sqrt{4 \pi D t}} \mathrm{e}^{-x^{2} /(4 D t)} \Theta(t)
$$

We consider travelling waves which satisfy $t_{n}=n \Delta$ so that the speed of threshold crossing events is given by $v=d / \Delta$. Moreover, we restrict attention to the case where $\Delta>\tau_{R}$ so that only one site is releasing $\mathrm{Ca}^{2+}$ at any one time. The, as yet, undetermined parameter $\Delta$ will be referred to as the period of a wave as it measures the time between successive release events that make up a saltatory travelling pulse. The saltatory wave is not periodic. Assuming that only the sites with index up to $N$ have crossed threshold we have that

$$
u(x, t)=\frac{\sigma}{\tau_{R}} \sum_{n=-\infty}^{N} \int_{0}^{\min \left(t-n \Delta, \tau_{R}\right)} \mathrm{d} t^{\prime} G\left(x-n d, t-t^{\prime}-n \Delta\right), \quad t>t_{N} .
$$

Since we are only interested in long time solutions that cause sites with increasing $n$ to cross threshold we consider the large $N$ limit and neglect all terms in the above sum with $n \leq 0$. It is convenient to introduce the following Laplace and Fourier transforms (which we distinguish from the untransformed functions by 
the use of their arguments):

$$
\begin{aligned}
u(k, t) & =\int_{-\infty}^{\infty} \mathrm{d} x \mathrm{e}^{-\mathrm{i} k x} u(x, t) \\
u(x, E) & =\int_{0}^{\infty} \mathrm{d} t \mathrm{e}^{-E t} u(x, t) .
\end{aligned}
$$

After Fourier and Laplace transforming (6) and performing the sum over sites we have that

$$
u(k, E)=\sigma \frac{G(k, E) \eta(E)}{\mathrm{e}^{\mathrm{i} k d+E \Delta}-1}
$$

where

$$
\eta(E)=\frac{1}{\sigma} \int_{0}^{\infty} \mathrm{d} t \mathrm{e}^{-E t} f(t)=\frac{\left[1-\mathrm{e}^{-E \tau_{R}}\right]}{E \tau_{R}} .
$$

Upon inverse transforming (9), we have the relation

$$
u(x+d, t+\Delta)-u(x, t)=\sigma H(x, t)
$$

where the inverse Laplace transform for calculating $H(x, t)$ may be taken either using a Bromwich integral or by using the convolution theorem for Laplace transforms. In either case one has that

$$
\begin{aligned}
H(x, t) & =\frac{1}{2 \pi i} \int_{c-\mathrm{i} \infty}^{c+\mathrm{i} \infty} G(x, E) \eta(E) \mathrm{e}^{E t} \mathrm{~d} E, \quad c>0 \\
& =\frac{1}{\tau_{R}} \int_{0}^{\tau_{R}} G\left(x, t-t^{\prime}\right) \mathrm{d} t^{\prime} .
\end{aligned}
$$

The integral representation (12) for $H(x, t)$ is particularly useful since it applies for any function $f(t)$ so that $\mathrm{Ca}^{2+}$ puff shapes other than the rectangular pulse shape (3) may be analysed. Throughout the rest of this paper we shall, however, focus upon the case where $f(t)$ is given by (3). Using (11) and initial data such that $u(0,0)=0$ it is simple to show that

$$
u(N d, N \Delta)=\sigma \sum_{n=1}^{N} H(n d, n \Delta) .
$$

Hence, one may determine the speed of the travelling wave in a self-consistent manner by demanding

$$
\lim _{N \rightarrow \infty} u(N d, N \Delta)=u_{c} .
$$

Note from (11) that waves do not propagate with an invariant shape even though the threshold crossing times occur on a regularly spaced temporal lattice. A saltatory travelling wave solution to the FDF model with an ionic pump $\left(\tau_{d}^{-1} \neq\right.$ 0 ), as analytically determined by equation [6], is illustrated in figure 1 . Note the large increase in the concentration of $\mathrm{Ca}^{2+}$ ions just after a release event. The 
saltatory nature of the wave may be directly attributed to the fact that release sites are not spread continuously throughout the system. Continuum models that neglect the discreteness of release sites are more appropriate for the study of travelling waves with constant profiles. For a recent study of the speed and stability of constant profile travelling waves in spatially extended continuum models of calcium release see the paper by Chopra et al. [1999].

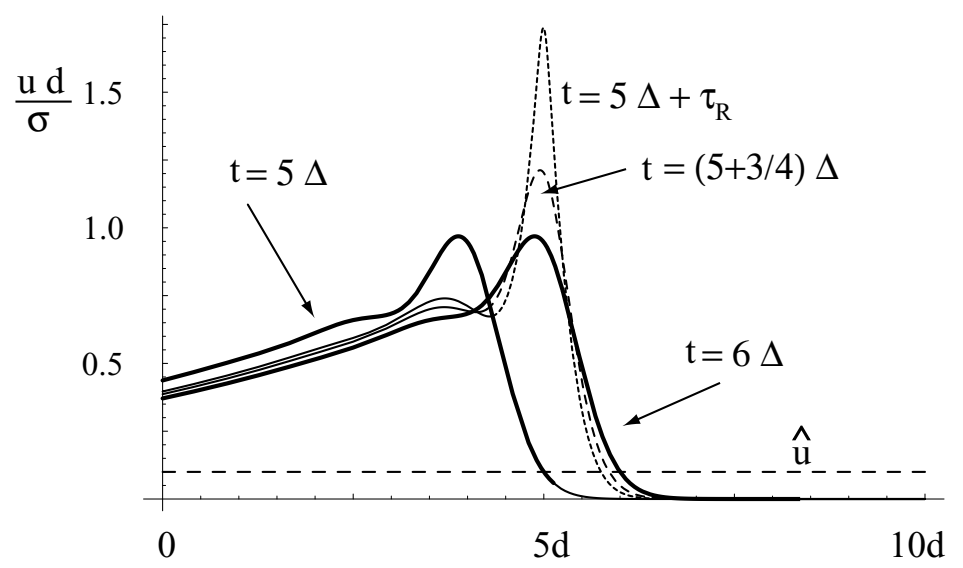

Figure 1: An example of a stable saltatory travelling wave analytically determined by equation (6) with $N=5$. The period $\Delta$ of the wave is determined self-consistently using the analysis presented in $\S 2$ as $\Delta=0.17$ for the choice of parameters $t_{d}=1, \tau_{D}=d^{2} / D=1, \tau_{R}=0.1$ and $\widehat{u}=u_{c} d / \sigma=0.1$

As an example of this method for determining the speed of travelling waves let us explore the limit as $\tau_{R} \rightarrow 0$. From (12) or 13 $H(x, t) \rightarrow G(x, t)$ so that the speed of the wave can be found by solving

$$
\widehat{u}=d \sum_{n=1}^{\infty} G(n d, n \Delta)=\sum_{n=1}^{\infty} \sqrt{\frac{\tau_{D}}{4 \pi n \Delta}} \exp \left(-n\left[\frac{\tau_{D}}{4 \Delta}+\frac{\Delta}{\tau_{d}}\right]\right) \equiv g(\Delta)
$$

where we have introduced the intersite diffusion time scale $\tau_{D}=d^{2} / D$ and the dimensionless threshold parameter $\widehat{u}=u_{c} d / \sigma$. In the limit $\tau_{d} \rightarrow \infty$ we recover the result presented by Pearson and Ponce Dawson [1998]. In this case $g(\Delta)$ is monotone in $\Delta$ and the speed of the travelling wave scales linearly as $D / d$. For finite $\tau_{d}$ the non-invertibility of $g(\Delta)$ allows the possibility of more than one solution. This then begs the question of stability. We deal with this issue in the next section. Suffice to say that, in general, for two co-existing solutions (with $\tau_{d}$ finite) it is always the faster of the two which is stable, whilst the slower is unstable.

In general the form of $H(x, t)$ is most easily calculated using the result that 
the Laplace transformed Green's function of the cable equation is given by

$$
G(x, E)=\frac{\mathrm{e}^{-\nu(E)|x|}}{2 D \nu(E)}, \quad \nu^{2}(E)=D^{-1}\left(\tau_{d}^{-1}+E\right) .
$$

Introducing the function $A(x, t)$ :

$$
A(x, t)=\frac{1}{2 D \tau_{R}} \frac{1}{2 \pi i} \int_{c-\mathrm{i} \infty}^{c+\mathrm{i} \infty} \frac{\mathrm{e}^{-\nu(E)|x|} \mathrm{e}^{E t}}{E \nu(E)} \mathrm{d} E .
$$

We may then write

$$
H(x, t)=A(x, t)-A\left(x, t-\tau_{R}\right) .
$$

One may evaluate $A(x, t)$ by closing the contour in the left hand complex plane and using a keyhole contour to cope with the branch cut of the function $\nu(E)$. The contour encloses the pole at 0 . It is then clear that $A(x, t)=R(x)-$ $Q(t) B(x, t)$ where

$$
R(x)=\frac{\sqrt{\tau_{d} D}}{2 D \tau_{R}} \exp \left(-\frac{|x|}{\sqrt{\tau_{d} D}}\right), \quad Q(t)=\frac{\mathrm{e}^{-t / \tau_{d}}}{D \tau_{R}}
$$

and

$$
B(x, t)=\frac{1}{2 \pi} \int_{-\infty}^{\infty} \frac{\mathrm{e}^{\mathrm{i} u|x|} \mathrm{e}^{-u^{2} D t}}{D u^{2}+\tau_{d}^{-1}} \mathrm{~d} u .
$$

After recognising 21] as an inverse Fourier transform with respect to the pseudoparameter $|x|$, one may use the convolution theorem for Fourier integrals to show that

$$
B(x, t)=\int_{-\infty}^{|x|} G\left(x^{\prime}, t\right) h\left(|x|-x^{\prime}, t\right) \mathrm{d} x^{\prime}+\int_{|x|}^{\infty} G\left(x^{\prime}, t\right) h\left(x^{\prime}-|x|, t\right) \mathrm{d} x^{\prime}
$$

where

$$
h(x, t)=\frac{R(x)}{Q(t)} .
$$

By performing the integrations in (22) $B(x, t)$ may be expressed in terms of the complementary error function:

$$
\operatorname{erfc}(x)=\frac{2}{\sqrt{\pi}} \int_{x}^{\infty} \mathrm{e}^{-u^{2}} \mathrm{~d} u
$$

as

$$
\begin{aligned}
B(x, t)=\frac{\sqrt{\tau_{d} D}}{4} \mathrm{e}^{t / \tau_{d}} & \left\{\exp \left(\frac{-|x|}{\sqrt{\tau_{d} D}}\right) \operatorname{erfc}\left(-\frac{|x|}{\sqrt{4 D t}}+\sqrt{\frac{t}{\tau_{d}}}\right)\right. \\
+ & \left.\exp \left(\frac{|x|}{\sqrt{\tau_{d} D}}\right) \operatorname{erfc}\left(\frac{|x|}{\sqrt{4 D t}}+\sqrt{\frac{t}{\tau_{d}}}\right)\right\} .
\end{aligned}
$$


In the limit $\tau_{d} \rightarrow \infty$ we have simply that

$$
A(x, t)=\frac{1}{2 D \tau_{R}}\left[\sqrt{\frac{4 D t}{\pi}} \exp \left(-\frac{x^{2}}{4 D t}\right)-|x| \operatorname{erfc}\left(\frac{|x|}{\sqrt{4 D t}}\right)\right] .
$$

In conjunction with [19], [14] and (15) this result forms the basis for the analysis presented by Ponce Dawson et al. [1999]. The more general analysis presented here allows one to go further and systematically analyse the effect of $\mathrm{Ca}^{2+}$ pumps and the shape of $\mathrm{Ca}^{2+}$ puffs on the speed of travelling waves. Furthermore, in the next section, we show how to analyse the stability of these travelling waves.

\subsection{Stability}

By regarding (2) as a map that generates the times of release events it has been demonstrated numerically Pearson and Ponce Dawson, 1998. Pearson et al., 1998, Keizer et al., 1998]) that in the limit $\tau_{d} \rightarrow \infty, \tau_{R} \rightarrow 0$ travelling wave states may lose stability via a sequence of period doubling bifurcations, ultimately leading to chaos. Such a numerical scheme is also possible within the generalised framework presented here (although we do not pursue this approach). However, the stability of solution branches to the speed equation 115 is readily obtained by perturbing the threshold crossing times according to $t_{n} \rightarrow t_{n}+\delta_{n}$ and examining the linearised evolution of the perturbations. Equivalently we could demand that

$$
\frac{u_{c}}{\sigma}=\sum_{n=1}^{\infty} H\left(n d, n \Delta+\delta_{n}\right) .
$$

After expanding to first order and considering perturbations of the form $\delta_{n}=\mathrm{e}^{n \lambda}$, $\lambda \equiv \alpha+\mathrm{i} \beta \in \mathbb{C},(\alpha, \beta \in \mathbb{R})$, we have simply that

$$
\Psi(\alpha, \beta) \equiv \sum_{n=1}^{\infty} \mathrm{e}^{n \alpha} \mathrm{e}^{\mathrm{i} n \beta} H^{\prime}(n d, n \Delta)=0
$$

where $H^{\prime}(x, t)=\partial H(x, t) / \partial t$. Differentiation of (12) shows that $H^{\prime}(x, t)=$ $\left[G(x, t)-G\left(x, t-\tau_{R}\right)\right] / \tau_{R}$. To find the stability of a wave as a function of system parameters one must solve $\operatorname{Re} \Psi(\alpha, \beta)=0$ and $\operatorname{Im} \Psi(\alpha, \beta)=0$ simultaneously for $\alpha$ and $\beta$ along the solution branch defined by 115 . For $\operatorname{Re}(\lambda)=\alpha<0$ the wave is considered to be linearly stable. The two possible types of bifurcation point where the system may lose stability are defined by the conditions $\alpha=0, \beta=0$ and $\alpha=0, \beta \neq 0$. For the first case $(\lambda \in \mathbb{R})$ a change in stability occurs when $\Psi(0,0)=0$. Moreover the stability of a branch on either side of the bifurcation point in the $\left(u_{c}, \Delta\right)$ plane is dependent upon the sign of $\mathrm{d} \Delta / \mathrm{d} u_{c}$. To establish this we implicitly differentiate the speed equation (15) with respect to $u_{c}$ to obtain

$$
\sigma \sum_{n=1}^{\infty} \frac{\partial H(n d, n \Delta)}{\partial \Delta}=\left(\frac{\mathrm{d} \Delta}{\mathrm{d} u_{c}}\right)^{-1}
$$


where

$$
\frac{\partial H(x, n \Delta)}{\partial \Delta}=n H^{\prime}(x, n \Delta)
$$

Expanding (28) for $\beta=0$ and $|\alpha| \ll 1$ and using the above two results we have that

$$
\alpha=-\frac{\sum_{n=1}^{\infty} H^{\prime}(n d, n \Delta)}{\sum_{n=1}^{\infty} n H^{\prime}(n d, n \Delta)}=-\sigma\left(\frac{\mathrm{d} \Delta}{\mathrm{d} u_{c}}\right) \Psi(0,0)
$$

It is possible to show by explicit construction that for $\tau_{R}=0$

$$
\Psi(0,0)=\sum_{n=1}^{\infty} G^{\prime}(n d, n \Delta)=\sum_{n=1}^{\infty} G(n d, n \Delta)\left[-\frac{1}{\tau_{d}}+\frac{\tau_{D}}{4 \Delta^{2}}-\frac{1}{2 n \Delta}\right]
$$

We see that in the limit $\tau_{D} \gg \tau$, where $\tau=2 \Delta\left(1-2 \Delta / \tau_{d}\right), \Psi(0,0)>0$ so that $\alpha<0$ when $\mathrm{d} \Delta / \mathrm{d} u_{c}>0$. As one moves into the opposite regime defined by $\tau_{D} \ll \tau$ we see from 32 that a solution may lose stability if $\mathrm{d} \Delta / \mathrm{d} u_{c}$ does not change sign.

The second type of instability arises when a complex eigenvalue crosses the imaginary axis. In this case $\Psi(0, \beta)=0$ has a solution for $\beta=\pi$ so that a change of stability occurs at the point in parameter space where $\sum_{n=1}^{\infty}(-1)^{n} H^{\prime}(n d, n \Delta)=$ 0 .

The stability of a solution branch is thus dependent upon whether or not either of the above two instabilities occurs.

\subsection{Bifurcation and stability diagrams}

In figure 2 we plot the period of a travelling wave as a function of the dimensionless threshold parameter $\widehat{u}=u_{c} d / \sigma$ for the special case that $\tau_{d}^{-1}=\tau_{R}=0$. As expected from (16) $\Delta$ is single-valued (since $g(\Delta)$ is monotone in $\Delta$ ) and increases with increasing $\widehat{u}$. No solutions are found beyond $\widehat{u} \sim 0.89$ (for $\tau_{D}=1$ ) in agreement with the original results of Pearson and Ponce Dawson [1998]. As discussed in $\S 2.1$ the solution can lose stability whenever $\Psi(0, \pi)=0$ or $\Psi(0,0)=0$. A plot of these two functions in figure 3 (along the solution branch for figure 2) shows that for increasing $\widehat{u}$ it is the former condition which is met first. A full numerical solution of $\Psi(\alpha, \beta)=0$ reveals that $\alpha<0$ for small $\widehat{u}$ so that the system first loses stability at around $\widehat{u} \sim 0.41$ (increasing $\widehat{u}$ ). In figure 4 we show how the period of the travelling wave changes as one introduces a non-zero value for $\tau_{d}^{-1}$, assuming instantaneous $\mathrm{Ca}^{2+}$ release $\left(\tau_{R}=0\right)$. For small $\tau_{d}^{-1}$ we recover the results described by figures 2 and 3 , namely that there is a single stable travelling wave (for a value of $\widehat{u}=0.1$ ). However, as $\tau_{d}^{-1}$ increases from zero one sees the appearance of another very slow unstable solution. The two solutions eventually coalesce at around $\tau_{d} \sim 1 / 3$, such that for smaller $\tau_{d}$ no solutions are possible, i.e. there is propagation failure for the type of saltatory travelling wave considered. It is precisely at this point in the bifurcation diagram 


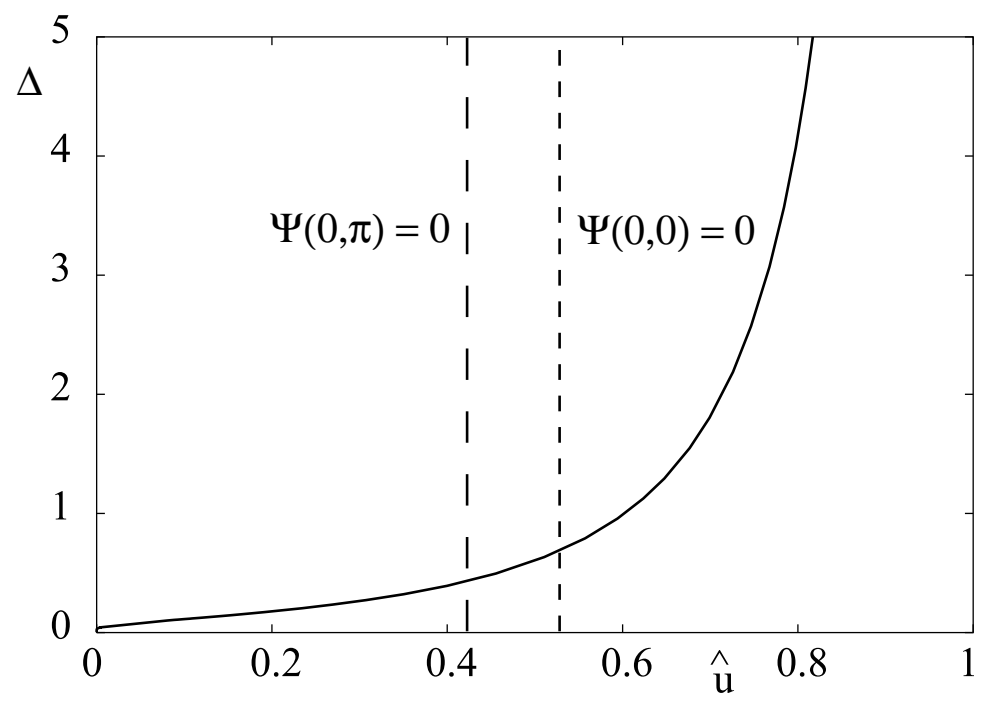

Figure 2: Period of a travelling wave as a function of $\widehat{u}$ for $\tau_{d}^{-1}=\tau_{R}=0$ and $\tau_{D}=1$. No solutions are found beyond $\widehat{u} \sim 0.89$. With increasing $\widehat{u}$ the system first loses stability where $\Psi(0, \pi)=0$.

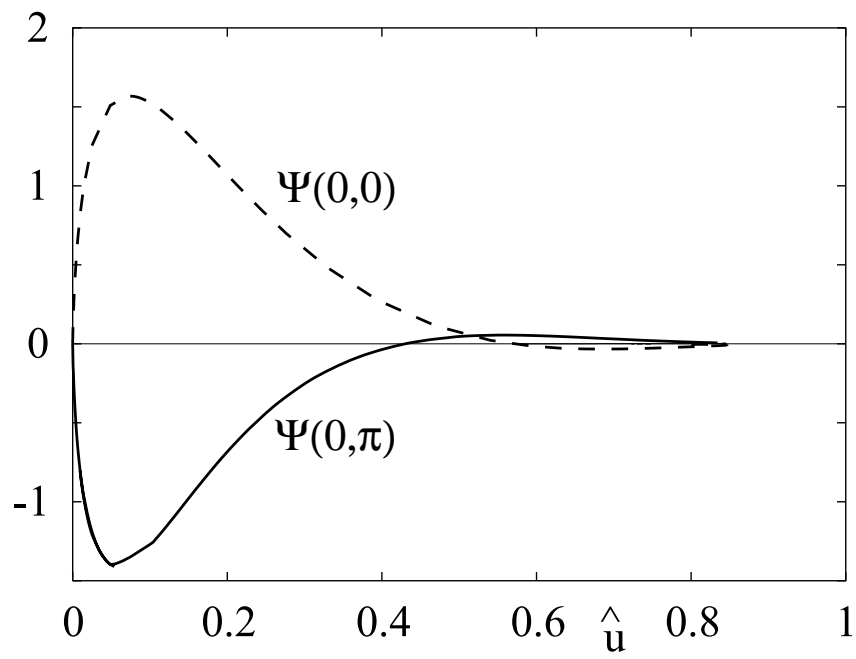

Figure 3: A plot of the functions $\Psi(0,0)$ and $\Psi(0, \pi)$ along the solution branch of figure 2 . The (single) solution loses stability at $\widehat{u} \sim 0.56$ via a real eigenvalue passing through zero and at $\widehat{u} \sim 0.41$ via a complex eigenvalue crossing the imaginary axis. A full solution of $\operatorname{Re} \Psi(\alpha, \beta)=0$ and $\operatorname{Im} \Psi(\alpha, \beta)=0$ shows that the solution branch is stable for small $\widehat{u}$ so that it first loses stability with increasing $\widehat{u}$ when $\Psi(0, \pi)=0$. 
that $\Psi(0, \beta)=0$. Hence, a change in stability of solution branches occurs at a limit point in the $\left(\tau_{d}^{-1}, \Delta\right)$ bifurcation diagram. One also sees that in this example (figure 4) that the period of the wave can almost double over the allowed range of values of $\tau_{d}$ for a stable travelling wave. To illustrate the effects of a

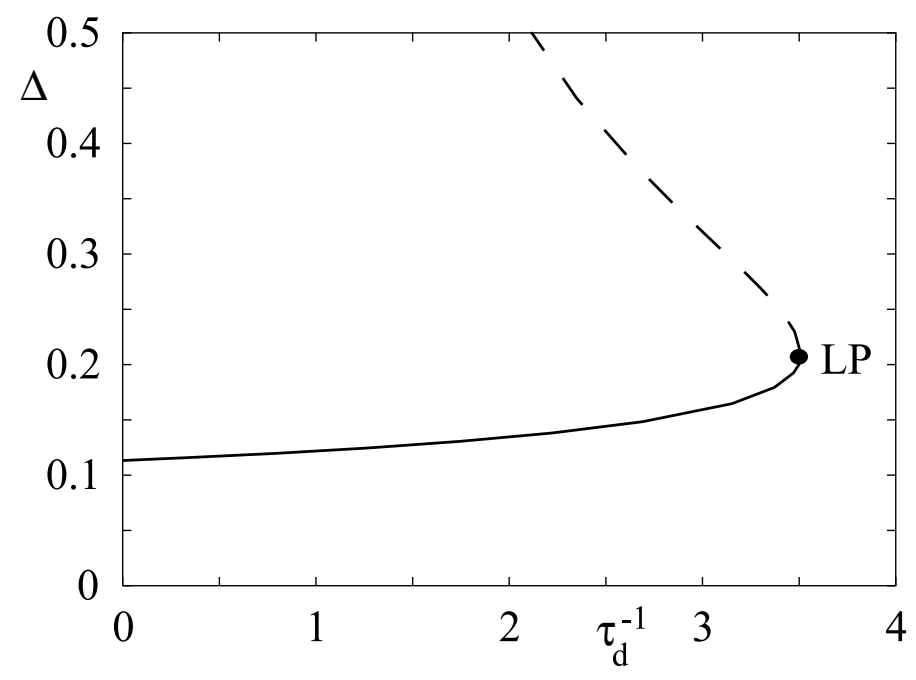

Figure 4: Solution branches in the $\left(\tau_{d}^{-1}, \Delta\right)$ with $\tau_{R}=0$ plane for $\widehat{u}=0.1$ and $\tau_{D}=1$. The limit point (LP) occurs when $\Psi(0, \pi)=0$. Solid (dashed) lines denote stable (unstable) solutions.

finite width for the calcium puff on the speed of the travelling wave we follow the limit point of the bifurcation diagram in figure 4 as a function of $\tau_{R}$. The results of this numerical continuation are shown in figure 5 . For $\tau_{R}<\Delta$ it can be seen that with increasing $\tau_{R}$ the limit point occurs at increasingly larger values of $\tau_{d}$. Hence, to avoid propagation failure (of the saltatory wave with single release events) for finite $\tau_{R}$ one must choose a correspondingly larger value for $\tau_{d}$ than one which ensures a stable travelling wave for the case with $\tau_{R}=0$.

\section{The continuum limit}

Until now we have focused upon saltatory travelling waves although in fact one of the major successes of the FDF model is to account for both saltatory and continuous waves. The continuous wave is unlike the saltatory one in that it travels with constant profile and is found in the limit of zero-lattice spacing. In comm on with the spike-diffuse-spike model presented by Coombes and Bressloff [2000], which also possesses constant profile travelling waves, it is a continuum model.

An analysis of the continuum FDF model may be obtained by combining the work of Ponce Dawson et al. [1999] (for the case $\tau_{d} \rightarrow \infty$ ) with the analysis 


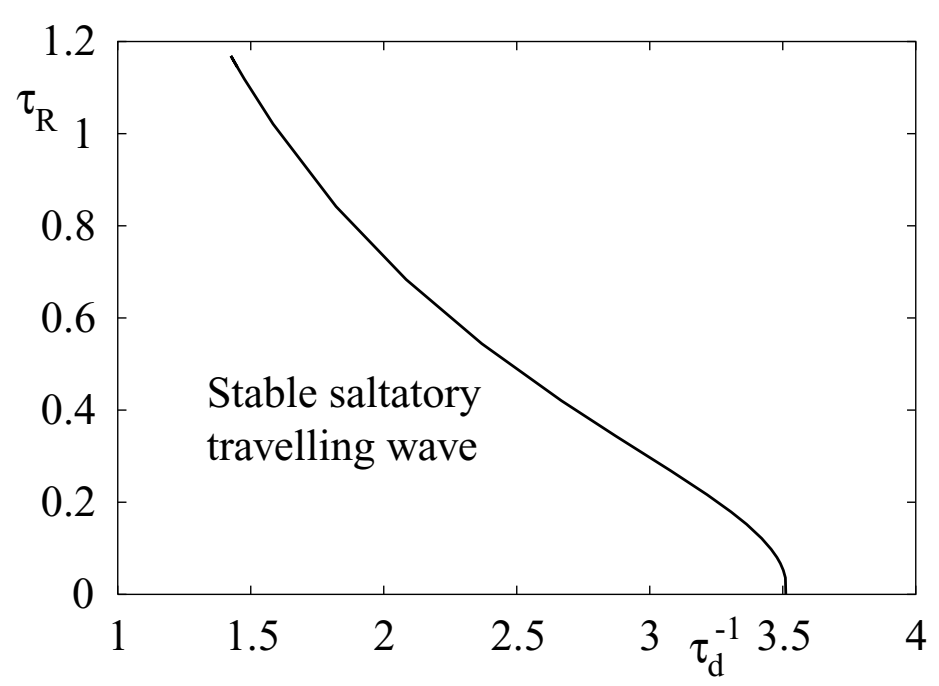

Figure 5: Continuation of the limit point (LP) (see figure 4) where $\Psi(0, \pi)=0$ in the $\left(\tau_{d}^{-1}, \tau_{R}\right)$ plane. For too small a value of $\tau_{d}$ and too large a value of $\tau_{R}$ propagation failure may result.

in this paper and considering the limit $d \rightarrow 0, \sigma \rightarrow 0$ and $\sigma / d \rightarrow$ constant. Alternatively, and more simply, one may analyse the continuum model directly. Denoting the first crossing of $u_{c}$ at position $x$ by $T(x)$ the continuum FDF model takes the form

$$
\frac{\partial u}{\partial t}=-\frac{u}{\tau_{d}}+D \frac{\partial^{2} u}{\partial x^{2}}+\rho(x) f(t-T(x)) .
$$

The function $\rho(x)$ describes the density distribution of the calcium sources. We consider the case of constant $\rho(x)$ and absorb this factor within the parameter $\sigma$. Travelling waves may be described with an ansatz of the form $T(x)=x / v$, where $v$ denotes the speed of the wave. In the travelling frame co-ordinate system $\xi \equiv v t-x$ the wave (with constant profile $u(\xi)$ ) is described with the second order ordinary differential equation:

$$
D u_{\xi \xi}-v u_{\xi}-\frac{u}{\tau_{d}}=-\frac{\sigma}{\tau_{R}} \Theta(\xi) \Theta\left(v \tau_{R}-\xi\right)
$$

where $u_{\xi} \equiv \mathrm{d} u / \mathrm{d} \xi$. For travelling pulse solutions which satisfy $\lim _{\xi \rightarrow \pm \infty} u(\xi)=0$ the solution to 34 takes the form

$$
u(\xi)= \begin{cases}\alpha_{1} \exp \left(m_{+} \xi\right) & -\infty<\xi<0 \\ \alpha_{2} \exp \left(m_{+} \xi\right)+\alpha_{3} \exp \left(m_{-} \xi\right)+\tau_{d} \sigma / \tau_{R} & 0<\xi<v \tau_{R} \\ \alpha_{4} \exp \left(m_{-} \xi\right) & \xi>v \tau_{R}\end{cases}
$$

with

$$
m_{ \pm}=\frac{1}{2 D}\left[v \pm \sqrt{v^{2}+4 D / \tau_{d}}\right]
$$


By ensuring the continuity of the solution and its first derivative at $\xi=0$ and $\xi=c \tau_{R}$ one may solve for the unknowns $\alpha_{1} \ldots \alpha_{4}$ as

$$
\begin{aligned}
\alpha_{1} & =\alpha_{3} \frac{m_{-}}{m_{+}}\left[1-\exp \left(-m_{+} v \tau_{R}\right)\right] \\
\alpha_{2} & =-\alpha_{3} \frac{m_{-}}{m_{+}} \exp \left(-m_{+} v \tau_{R}\right) \\
\alpha_{3} & =\frac{\tau_{d} \sigma}{\tau_{R}} \frac{m_{+}}{\left(m_{-}-m_{+}\right)} \\
\alpha_{4} & =\alpha_{3}\left[1-\exp \left(-m_{-} v \tau_{R}\right)\right] .
\end{aligned}
$$

The self-consistent speed of the travelling wave may be determined by demanding that $u(x, x / v)=u_{c}$. In the travelling frame co-ordinate this reduces to $u_{c}=u(0)$, so that from 35 the speed of the travelling pulse satisfies

$$
\widetilde{u} \equiv \frac{u_{c} \tau_{R}}{\sigma \tau_{d}}=\frac{m_{-}}{m_{-}-m_{+}}\left[1-\mathrm{e}^{-m^{+} v \tau_{R}}\right] .
$$

It is straight-forward to show from 441 that for $\widetilde{u}<1$ the velocity $v$ scales as $\sqrt{D / \tau_{d}} \sqrt{1 / \widetilde{u}-1}$ for large $v$. For $\tau_{d}^{-1}=0, v$ scales as $\sqrt{D / \tau_{R}} \sqrt{\sigma / u_{c}}$.

\subsection{Stability}

For the purposes of a linear stability analysis it is more convenient to work in terms of the original variables $(x, t)$ rather than in the moving frame. In this case the formal solution to (33) can be expressed as

$$
\begin{aligned}
u(x, t) & =\int_{-\infty}^{t} \mathrm{~d} s \int_{-\infty}^{\infty} \mathrm{d} y G(x-y, t-s) f(s-y / v) \\
& =\frac{\sigma}{\tau_{R}} \int_{-\infty}^{v\left(t-\tau_{R}\right)} \mathrm{d} y \int_{y / v}^{y / v+\tau_{R}} \mathrm{~d} s G(x-y, t-s) .
\end{aligned}
$$

Using the following Fourier integral representation for the Green's function of the cable equation:

$$
G(x, t)=\int_{-\infty}^{\infty} \frac{\mathrm{d} k}{2 \pi} \mathrm{e}^{\mathrm{i} k x} \mathrm{e}^{-\epsilon(k) t}, \quad \epsilon(k)=-\frac{1}{\tau_{d}}+D k^{2}
$$

the solution $u(x, t)$ takes on the compact form

$$
u(x, t)=\sigma \int_{-\infty}^{\infty} \frac{\mathrm{d} k}{2 \pi} \mathrm{e}^{\mathrm{i}(t-x / v)} \frac{\eta(\mathrm{i} k)}{\epsilon(k / v)+\mathrm{i} k}
$$

which may be evaluated using contours in the lower and upper half complex plane and is equivalent to (35). It is natural to consider local perturbations of the firing times given by $T(x)=x / v+g(x)$. For convenience we introduce the difference between perturbed and unperturbed trajectories as $\delta_{g} u(x, T(x))=$ 
$u(x, T(x)+g(x))-u(x, T(x))$. To first order in the perturbations we have from (42) that

$$
\delta_{g} u(x, T(x))=\int_{-\infty}^{x / v} \mathrm{~d} s \int_{-\infty}^{\infty} \mathrm{d} y G(x-y, x / v-s) f^{\prime}(s-y / v)[g(x)-g(y)] .
$$

The stability of solutions is determined by demanding that $\delta_{g} u(x, T(x))=0$ for all $x$. For solutions of the form $g(x)=\mathrm{e}^{\lambda x}$ this yields the characteristic equation $I(\lambda)-I(0)=0$, where

$$
I(\lambda)=\sigma \int_{-\infty}^{\infty} \frac{\mathrm{d} k}{2 \pi} \frac{\mathrm{i} k \eta(\mathrm{i} k)}{\epsilon(k / v+\mathrm{i} \lambda)+i k} .
$$

Asymptotic stability holds if all non-zero solutions of the characteristic equation have negative real part. (The existence of a solution $\lambda=0$ reflects the translation invariance of the underlying dynamical system). Equation (46) can be evaluated by closing the contour in the lower-half complex $k$-plane. Since $f(t)=0$ for $t<0$ it follows that any poles of $\eta(i k)$ lie in the upper-half complex plane so that we only have to consider poles arising from the zeros of the function $\epsilon(k / v+\mathrm{i} \lambda)+\mathrm{i} k$. The latter are given explicitly by $k=i k_{ \pm}(\lambda)$ where

$$
\frac{k_{ \pm}(\lambda)}{v}=-\left(\lambda+\frac{v}{2 D}\right) \pm \sqrt{R(\lambda)}
$$

with

$$
R(\lambda)=v^{2} /\left(4 D^{2}\right)+c \lambda / D+1 /\left(D \tau_{d}\right)
$$

Let us decompose $\lambda$ into real and imaginary parts according to $\lambda=\alpha+i \beta$. Then

$$
k_{ \pm}(\lambda)=-u_{ \pm}(\alpha, \beta)-i v_{ \pm}(\alpha, \beta)
$$

with

$$
\frac{u_{ \pm}(\alpha, \beta)}{v}=\alpha+\frac{v}{2 D} \mp A(\alpha, \beta), \quad \frac{v_{ \pm}(\alpha, \beta)}{v}=\beta \mp B(\alpha, \beta)
$$

and (for $\beta>0$ )

$$
\begin{aligned}
& A(\alpha, \beta)=\sqrt{\frac{1}{2}\left[R(\alpha)+\sqrt{R(\alpha)^{2}+c^{2} \beta^{2} / D^{2}}\right]} \\
& B(\alpha, \beta)=\sqrt{\frac{1}{2}\left[-R(\alpha)+\sqrt{R(\alpha)^{2}+c^{2} \beta^{2} / D^{2}}\right]} .
\end{aligned}
$$

In order to check stability of a solitary pulse we need to establish that there are no solutions for which $\alpha>0$. We distinguish two cases according to the sign of $u_{+}(\alpha, \beta)$.

Case A: $u_{+}(\alpha, \beta)<0$ so that the only pole in the lower-half complex plane is at $i k_{-}(\lambda)$ and $I(\lambda)=I_{-}(\lambda)$ where we define

$$
I_{ \pm}(\lambda)=\frac{\sigma v^{2}}{\tau_{R}} \frac{\mathrm{e}^{k_{ \pm}(\lambda) \tau_{R}}-1}{k_{+}(\lambda)-k_{-}(\lambda)} .
$$


Case B: $u_{+}(\alpha, \beta)>0$ so that both poles contribute and $I(\lambda)=I_{-}(\lambda)-I_{+}(\lambda)$. One may then determine the stability of solution branches by simultaneously solving $\operatorname{Re} I(\lambda)-\operatorname{Re} I(0)=0$ and $\operatorname{Im} I(\lambda)-\operatorname{Im} I(0)=0$ for $\alpha$ and $\beta$ where

$$
\begin{aligned}
& \operatorname{Re} I_{ \pm}(\lambda)=\frac{\sigma v}{2 \tau_{R}} \frac{\mathrm{e}^{-u_{ \pm} \tau_{R}} \cos \left(v_{ \pm} \tau_{R}\right)-1}{A^{2}+B^{2}} \\
& \operatorname{Im} I_{ \pm}(\lambda)=-\frac{\sigma v}{2 \tau_{R}} \frac{\mathrm{e}^{-u_{ \pm} \tau_{R}} \sin \left(v_{ \pm} \tau_{R}\right)}{A^{2}+B^{2}} .
\end{aligned}
$$

\subsection{Bifurcation and stability diagrams}

In figure 6 we plot the speed of the constant profile travelling pulse as a function of the dimensionless threshold parameter $\widetilde{u}=u_{c} \tau_{R} /\left(\sigma \tau_{d}\right)$. In common with the lattice FDF model one sees the co-existence of two travelling waves with speeds that approach each other as $\widetilde{u}$ increases. Eventually the two waves coalesce at a limit point in the $(\widetilde{u}, v)$ bifurcation diagram such that propagation failure can result for too large a choice of the threshold parameter. A numerical solution of the characteristic equation $I(\alpha+\mathrm{i} \beta)-I(0)=0$ for $\alpha$ and $\beta$ shows that it is the faster of the two branches that is stable $(\alpha<0)$. The change in stability at the limit point in the $(\widetilde{u}, v)$ plane occurs when a real eigenvalue crosses through zero $(\beta=0)$. In figure 7 we show a numerical continuation of the limit point in

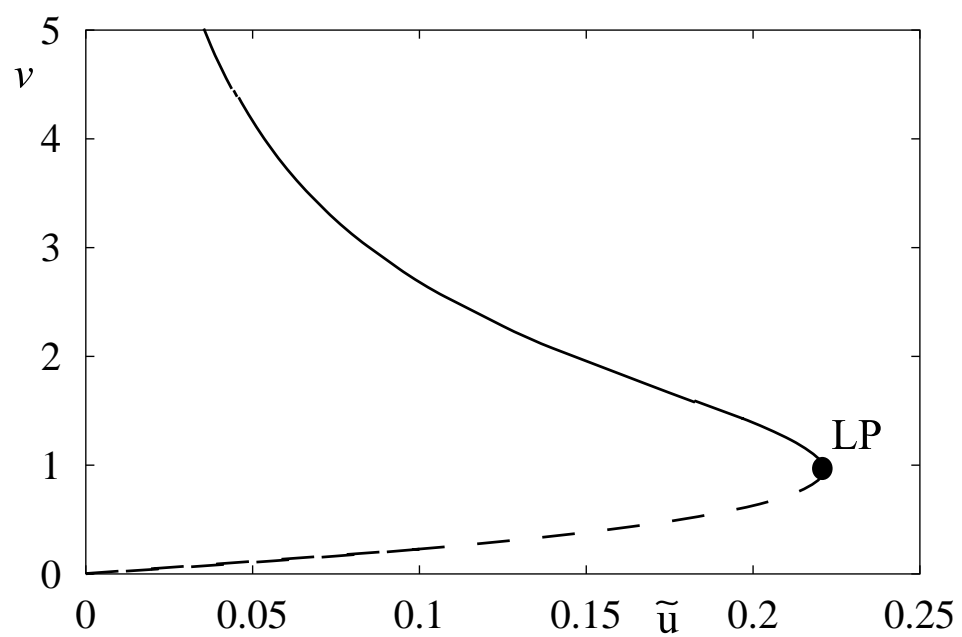

Figure 6: Speed $v$ as a function of the dimensionless threshold parameter $\widetilde{u}$ in the continuum FDF model for $\tau_{d}=\tau_{R}=D=1$. A linear stability analysis shows that it is the faster of the two branches that is stable.

figure 6 (the point where the two solutions coalesce). We draw the conclusion that propagation failure of a constant profile travelling pulse is less likely for the case of temporally extended $\mathrm{Ca}^{2+}$ puffs (large $\left.\tau_{R}\right)$. 


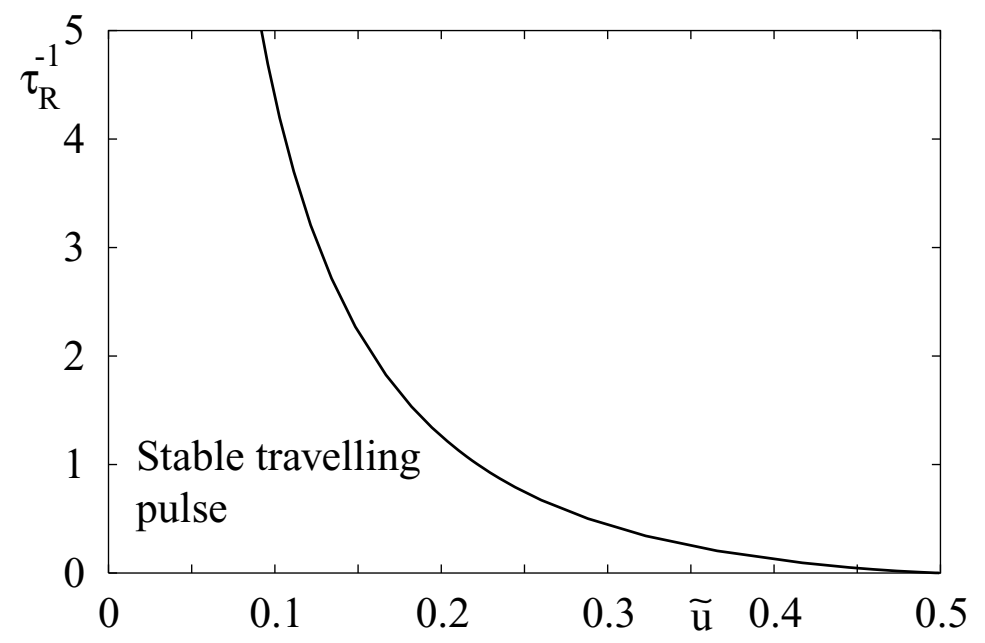

Figure 7: Continuation of the limit point (LP) shown in figure 6 in the $\left(\widetilde{u}, \tau_{R}^{-1}\right)$ parameter plane. For too small a value of $\tau_{R}$ and too large a value of $\widetilde{u}$ propagation failure may result.

\section{Discussion}

In this paper we have used an exact mathematical treatment to focus upon the effects of pumps that move $\mathrm{Ca}^{2+}$ ions from the cytosol to the endoplasmic reticulum within the FDF model of $\mathrm{Ca}^{2+}$ release. The presence of such pumps was shown to lead to the co-existence of a fast and slow travelling wave. By considering perturbations in the times of release events we were able to formulate a condition for linear stability which predicts that it is always the faster of the two that is stable. Using the exact derived form for the wave speed as a function of system parameters also allowed us to show that pumps operating above some critical rate (for a given duration and strength of $\mathrm{Ca}^{2+}$ puff) can effectively block the propagation of single pulse travelling waves. Moreover, we have highlighted the differences between a discrete and continuous distribution of release sites. In the former case travelling waves propagate with a non constant profile and the speed of the wave scales with the diffusion coefficient of the FDF model, whilst in the latter case waves move with constant profile at a speed that scales with the square-root of the diffusion coefficient. The detailed dependence of wave speed upon the pump rate, duration and strength of $\mathrm{Ca}^{2+}$ puffs and the regular spacing between release sites has also been described. In the limit of infinitely slow pumps $\left(\tau_{d}^{-1} \rightarrow 0\right)$ we recover results originally discovered in Keizer et al., 1998, Pearson and Ponce Dawson. 1998. Ponce Dawson et al.. 1999]. We end this section with a few points regarding how best to extend the work in this paper.

Firstly, the exponential decay discussed by Keizer et al. [1998] and Pearson and Ponce Dawson [1998] to mimic ionic pumps is at best a crude approximation of the way real pumps operate. The main difference between the decay term considered in this paper and biological pumps is that the latter saturate. A common 
model of such a process is to consider a sink of the form $u^{n} /\left(K+u^{n}\right)$ (for a further discussion of how one might model $\mathrm{Ca}^{2+}$ pumps see Li et al., 1995]). A piecewise linear version of such a function may be studied with the formalism developed here. It is likely that the stability boundaries would be shifted with propagation favoured over propagation failure since the pumps have a maximal rate that is essentially independent of the $\mathrm{Ca}^{2+}$ concentration. Secondly, the FDF model ignores the effects of multiple $\mathrm{Ca}^{2+}$ puffs at release sites. To generalise the model one might consider the replacement $f\left(t-t_{n}\right) \rightarrow \sum_{m} f\left(t-t_{n}^{m}\right)$ where $t_{n}^{m}$ is the $m$ th time that the concentration at site $n$ crosses the threshold $u_{c}$ from below. Of course in this case one must also be careful to model the refractory period of a release event. Assuming this is handled properly it would then be possible to study the class of periodic travelling waves defined by $t_{n}^{m}=(n+m k) \Delta$. An extension of the techniques presented in this paper might be used to determine a self-consistency condition for the existence of such travelling waves. One may then construct the dispersion relation for the period $\Delta=\Delta(k)$. Moreover, with an appropriate ansatz for the threshold crossing times, the description of circular waves, spirals and scrolls in the two and three dimensional generalisations of the FDF may also be possible. The extension of the FDF model also allows the possibility of irregular wave trains. To analyse non-periodic waves where $\mathrm{Ca}^{2+}$ puffs travel as an irregularly spaced train one could formulate a kinematic theory of wave propagation based upon the analysis of the periodic travelling waves [Rinzel and Maginu, 1984]. Such a formulation would attempt to follow the progress of individual release events without regards to the detailed structure of the calcium puffs. Thirdly, calcium is heavily buffered in all cells with about $99 \%$ of the available $\mathrm{Ca}^{2+}$ bound to large proteins. The presence of $\mathrm{Ca}^{2+}$ buffers changes the nature of the $\mathrm{Ca}^{2+}$ transport equations and hence the properties of travelling waves. Indeed for buffers with fast kinetics it may be more appropriate to model the transport of $\mathrm{Ca}^{2+}$ ions with a nonlinear diffusion-advection equation where the advection is the result of $\mathrm{Ca}^{2+}$ transport by a mobile buffer Wagner and Keizer, 1994, Sneyd et al., 1998). Interestingly in the two variable reduction of the De Young-Keizer model (for calcium waves) with $\mathrm{Ca}^{2+}$ buffering, numerical simulations produce nonlinear wave trains whose speed increases linearly with time [Jafri and Keizer, 1997]. Finally it is important to acknowledge that real biological systems have some element of disorder in the spatial distribution of calcium relegse sites throughout a cell. Recent numerical experiments by Bugrim et al. [1997] suggest that the propagation of (calcium) waves in a cell with randomly distributed release sites is reminiscent of that seen in forest fire models, flame propagation in random materials and epidemic spread. Together with a numerical study of the solutions that arise when travelling waves cease to exist or lose stability, all of the above are topics that may be pursued within the framework of the FDF model. 


\section{Acknowledgments}

I would like thank Markus Owen for a careful reading of this manuscript as well as the referees for their helpful comments. This work was supported in part by a grant from the Nuffield foundation.

\section{References}

M J Berridge. Elementary and global aspects of calcium signalling. Journal of Neurophysiology, 499:291-306, 1997.

M J Berridge. Neuronal calcium signalling. Neuron, 21:13-26, 1998.

M J Berridge and G Dupont. Spatial and temporal signalling by calcium. Current Opinion in Cell Biology, 6:267-274, 1994.

M D Bootman, M J Berridge, and P Lipp. Cooking with calcium: The recipes for composing global signals from elemenatry events. Cell, 91:367-373, 1997.

E A Bugrim, Zhabotinsky, and I R Epstein. Calcium waves in a model with a random spatially discrete distribution of $\mathrm{Ca}^{2+}$ release sites. Biophysical Journal, 73:2897-2906, 1997.

N Callamaras, J S Marchant, X P Sun, and I Parker. Activation and coordination of $\operatorname{InsP}(3)$-mediated elementary $\mathrm{Ca}^{2+}$ events during global $\mathrm{Ca}^{2+}$ signals in Xenopus oocytes. Journal of Physiology, 509:81-91, 1998.

G C Chopra, B D Sleeman, J Brindley, D G Knapp, and A V Holden. Velocity and stability of solitary planar travelling wave solutions of intracellular $\left[\mathrm{Ca}^{2+}\right]$. Bulletin of Mathematical Biology, 61:273-301, 1999.

S Coombes and P C Bressloff. Solitary waves in a model of dendritic cable with active spines. SIAM Journal on Applied Mathemaics (to appear), 2000.

G W De Young and J Keizer. A single pool $\mathrm{IP}_{3}$-receptor based model for agonist stimulated $\mathrm{Ca}^{2+}$ oscillations. Proceedings of the National Academy of Sciences USA, 89:9895-9899, 1992.

G DuPont and A Goldbeter. Properties of intracellular $\mathrm{Ca}^{2+}$ waves generated by a model based on $\mathrm{Ca}^{2+}$-induced-Ca ${ }^{2+}$ release. Biophysical Journal, 67: 2191-2204, 1994.

A Goldbeter. Biochemical Oscillations and Cellular Rhythms. Cambridge University Press, 1996.

M S Jafri and J Keizer. On the roles of $\mathrm{Ca}^{2+}$ diffusion, $\mathrm{Ca}^{2+}$ buffers, and the endoplasmic reticulum in $\mathrm{IP}_{3}$-induced $\mathrm{Ca}^{2+}$ waves. Biophysical Journal, 69: 2139-2153, 1995. 
M S Jafri and J Keizer. Agonist-induced calcium waves in oscillatory cells: A biological example of Burgers' equation. Bulletin of Mathematical Biology, 59: 1125-1144, 1997.

J E Keizer, G D Smith, S Ponce Dawson, and J Pearson. Saltatory propagation of $\mathrm{Ca}^{2+}$ waves by $\mathrm{Ca}^{2+}$ sparks. Biophysical Journal, 75:595-600, 1998.

Y X Li, J Keizer, S S Stojilkovic, and J Rinzel. $\mathrm{Ca}^{2+}$ excitability of the ER membrane: an explanation for $\mathrm{IP}_{3}$-induced $\mathrm{Ca}^{2+}$ oscillations. American Journal of Physiology, 269:C1079-92, 1995.

I Parker and Y Yao. Regenerative release of calcium from functionally discrete subcellular stores by inisitol trisphosphate. Proceedings of the Royal Society London B, 246:269-274, 1991.

J E Pearson and S Ponce Dawson. Crisis on skid row. Physica A, 257:141-148, 1998.

J E Pearson, S Ponce Dawson, J Keizer, and G Smith. Crisis on skid row. http://www-xdiv.lanl.gov/XCM/pearson/skid_row_relay.ps. 1998.

S Ponce Dawson, J Keizer, and J E Pearson. Fire-diffuse-fire model of dynamics of intracellular calcium waves. Proceedings of the National Academy of Sciences USA, 96:6060-6063, 1999.

J Rinzel and K Maginu. Non-equilibrium Dynamics in Chemical Systems, chapter Kinematic analysis of wave pattern formation in excitable media, pages 107-113. Springer-Verlag, 1984.

T A Rooney and A P Thomas. Intracellular calcium waves generated by Ins $(1,4,5) \mathrm{p}_{3}$-dependent mechanisms. Cell Calcium, 14:674-690, 1993.

J Sneyd, P D Dale, and A Duffy. Traveling waves in buffered systems: applications to calcium waves. SIAM Journal on Applied Mathematics, 58:1178-1192, 1998.

J Sneyd, S Girard, and D Clapham. Calcium wave propagation by calciuminduced-calcium release: an unusual excitable system. Bulletin of Mathematical Biology, 55:315-344, 1993.

J Wagner and J Keizer. Effects of rapid buffers on $\mathrm{Ca}^{2+}$ diffusion and $\mathrm{Ca}^{2+}$ oscillations. Biophysical Journal, 67:447-456, 1994. 